\section{An Evaluation of Two Seedling Phenotyping Protocols to Assess pH Adaptability in Deciduous Azalea (Rhododendron sect. Pentanthera G. Don)}

\author{
Alexander Q. Susko \\ Department of Horticultural Science, University of Minnesota, 1970 Folwell \\ Avenue, St. Paul, MN 55108
}

Timothy A. Rinehart

USDA-ARS Thad Cochran Southern Horticultural Research Laboratory, P.O. Box 287, Poplarville, MS 39470

\section{James M. Bradeen}

Department of Plant Pathology, The Stakman-Borlaug Center for Sustainable Plant Health, 1991 Upper Buford Circle, St. Paul, MN 55108

\section{Stan C. Hokanson ${ }^{1}$ \\ Department of Horticultural Science, University of Minnesota, 1970 Folwell Avenue, St. Paul, MN 55108}

Additional index words. iron deficiency chlorosis, high-throughput phenotyping, rhizosphere acidification, abiotic stress tolerance, Ericaceae

\begin{abstract}
Deciduous azaleas are an important element of residential and commercial landscapes in the United States after substantial trait improvements to increase their market appeal. Despite progress in breeding for ornamental characteristics and cold hardiness, intolerance to elevated $\mathrm{pH}$ and calcareous soils continues to limit their use in managed landscapes. Therefore, we assessed the utility of in vitro and greenhouse phenotyping approaches to evaluate and select for improved soil $\mathrm{pH}$ tolerance to increase the efficiency of breeding for this important trait. The research presented offers an example for implementing image-based phenotyping to expedite cultivar development in woody ornamental crops.
\end{abstract}

Deciduous azalea (Rhododendron sect. Pentanthera G. Don) cultivars and species are highly ornamental shrubs that are valued in northern landscapes for their colorful and prolific flowers. One important group of deciduous azalea cultivars is the Northern Lights series, which since 1979 has grown to 15 interspecific hybrids including the original 'Northern Lights', 'Mandarin Lights', and 'Lemon Lights' that bring vivid pink, orange, and yellow colors into U.S.

\footnotetext{
Received for publication 26 Sept. 2017. Accepted for publication 21 Nov. 2017.

We thank Alan Smith for permission to use tissue culture lab space and equipment. We also thank Elsa Eschenaur and Mitchal Peterson for their assistance in subculturing and help with other tissue culture lab techniques presented in this research. Mention of trade names or commercial products in this publication is solely for the purpose of providing specific information and does not imply recommendation or endorsement by the U.S. Department of Agriculture. USDA is an equal opportunity provider and employer.

${ }^{1}$ Corresponding author. E-mail: hokan017@umn. edu.
}

Department of Agriculture (USDA) hardiness Zone 4 northern landscapes (Hokanson, 2010). With nearly 630,000 plants sold at a combined wholesale value of $\$ 1.02$ million between 2014 and 2016, these and other deciduous azalea cultivars represent a reliable source of revenue for woody plant growers who cater to landscape markets in cold climates (L. Caton, Sidhu and Sons, personal communication). Given the series' popularity, the University of Minnesota breeding program continues to expand the series, with the first true red 'UMNAZ633' Electric Lights ${ }^{\mathrm{TM}}$ Red and double-flowered 'UMNAZ493' Electric Lights $^{\mathrm{TM}}$ Double Pink introductions bringing new ornamental characteristics to cold-hardy woody ornamental germplasm (Hokanson et al., 2015).

Progenitors of the cultivars mentioned previously primarily resulted from crosses between North American deciduous azalea species native to the southern and eastern United States with European deciduous azalea hybrids (Mollis Azalea, Exbury, and Knapp Hill hybrids) from the early 20th century (Moe and Pellett, 1986). Each of these native species contributes unique ornamental attributes to cultivars released. These include pink flowers from wild-collected Rhododendron prinophyllum (Small) Millais and large, durable, orange flowers from Rhododendron calendulaceum (Michx.) Torr. (Moe and Pellett, 1986; Widrlechner, 1982). Additional ornamental characteristics such as fragrance from Rhododendron atlanticum (Ashe) Rheder and white flowers from Rhododendron viscosum (L.) Torr. were also introgressed into the breeding germplasm (Hokanson, 2010). The combination of traits from each of these species and their segregation in seedling populations has enabled a diverse series of Rhododendron cultivars to be developed for northern areas of the United States, where cultivation of the genus was historically thought to be impossible because of extreme winter cold temperatures (Widrlechner, 1982).

The greatest systematic breeding effort has been focused on improving the cold hardiness of floral and vegetative tissues (Moe and Pellett, 1986). Initial cold hardiness evaluations required substantial screening time in the field as the initial parental germplasm and progeny evaluations that led to 'Northern Lights' spanned the years 195779, a 22-year period. These initial efforts resulted in a series of deciduous azalea cultivars that are reliably cold-hardy in USDA Zone 4 (Hokanson, 2010). As cultivation of deciduous azaleas has spread throughout the Midwestern United States, tolerance to high $\mathrm{pH}$ or calcareous soils (calcium carbonate, $\mathrm{CaCO}_{3}$ ) that predominate in the region has become the most pressing trait to improve. High-pH soils severely limit deciduous azalea growth, primarily by restricting micronutrient availability, such as iron, for uptake by roots (Brady and Weil, 2004). Bicarbonate ions present in calcareous soils raise soil $\mathrm{pH}$ and keep the soil buffered, resulting in slower acidification of soil, further limiting micronutrient availability (Brady and Weil, 2004). This reduction in iron uptake on high-pH or calcareous soils leads to chlorosis and decreased photosynthetic capacity, which over time reduces vigor and increases mortality of deciduous azaleas in the landscape (Galle, 1974). Improving adaptability to high-pH or calcareous soils (henceforth referred to as $\mathrm{pH}$ adaptability) of cultivars would reduce plant maintenance and open up new markets in continental climates characterized by calcareous or high$\mathrm{pH}$ soils.

Although some systematic selection against iron deficiency chlorosis has occurred in evergreen Rhododendron, the variation is hard to phenotype consistently because of confounding nutrient deficiencies and measurement error using a qualitative (1-10) rating scale in breeding populations (Dunemann et al., 1999; Preil and Ebbinghaus, 1994). Furthermore, prospects for improving $\mathrm{pH}$ adaptability in deciduous azalea are slowed by long generation times and limited timeframe (at leaf flush) for observing symptoms of high $\mathrm{pH}$ stress such as iron deficiency chlorosis. Like many woody ornamentals, deciduous azaleas take 
between 8 and 10 months from crossing to seed germination, and an additional 2 years before plants reach maturity for field trials (Susko, 2016). Growing plants in containers before planting in the field requires space and labor that is often a limiting factor in determining how many seedlings can be produced for screening each season. Therefore, selection at the early seedling stage could both reduce the number of plants carried forward and expedite breeding deciduous azaleas.

Rather than waiting for the manifestation of chlorosis in whole plant experiments, characterization of the root-soil interface (rhizosphere), including localized reduction in $\mathrm{pH}$ (rhizosphere acidification), could more quickly indicate whether certain genotypes will successfully acquire micronutrients and mitigate symptoms of high $\mathrm{pH}$ stress (Guerinot and Yi, 1994). Known as Strategy 1 iron acquisition, dicot plants extrude protons into the rhizosphere to create favorable conditions for iron solubility and reduction that enable its translocation into root cells (Brady and Weil, 2004; Briat and Lobréaux, 1997). Direct acidification of the rhizosphere by proton extrusion by plasma membrane $\mathrm{H}^{+}$-ATPase is a well-documented response to iron deficiency in dicot plants (Yi and Guerinot, 1996). Indirect acidification of the rhizosphere through other nutrient exchanges such as nitrogen is also possible (Haynes, 1990). Specifically, ammonium uptake also occurs in concert with proton extrusion, and thus, results in acidification of the rhizosphere (Escobar et al., 2006). Regardless of the exact mechanism by which rhizosphere acidification is initiated, quantifying phenotypic variation in rhizosphere acidification capability could facilitate identification of selections capable of acquiring sufficient iron to promote healthy growth under calcareous soil conditions.

Given the quantitative nature of $\mathrm{pH}$ adaptability identified in other plants (Froechlich and Fehr, 1981; Gogorcena et al., 2001; Gonzalo et al., 2011), the use of imagebased phenotyping methods could improve our ability to more quickly and more precisely identify selectable genetic variation for traits such as rhizosphere acidification that play a role in $\mathrm{pH}$ adaptability. Image-based phenotyping protocols are potentially well suited to ameliorating the problems (i.e., subjectivity, slow speed) inherent in phenotyping $\mathrm{pH}$ adaptability. Image-based screening approaches have been used in many other crops to improve quantification of challenging traits, including root architecture, water use efficiency, and nutrient deficiencies while maintaining yield goals (Berger et al., 2010; Clark et al., 2013; Shi et al., 2013). Systematic phenotyping protocols could then be used to identify superior genotypes within existing breeding germplasm or in wild populations to identify new sources of tolerance for elevated $\mathrm{pH}$ for deciduous azaleas.

To address the challenges of identifying $\mathrm{pH}$ adaptability in deciduous azaleas, we evaluated an in vitro phenotyping protocol to identify quantitative variation in $\mathrm{pH}$ change in the root rhizosphere. We focused our screening methods on in vitro plants in an effort to increase the efficiency of breeding elevated $\mathrm{pH}$-tolerant plants. We tested seedlings from the same crosses in vitro using $\mathrm{pH}$ indicator dye to measure plant-induced changes to the culture medium $\mathrm{pH}$ in the presence of liming treatments. We also tested seedlings from the same families in the greenhouse to measure leaf area change in response to liming treatments. Leaf area was quantified using image analysis methods implemented in MATLAB (MathWorks, Inc., Natick, MA) and ImageJ (National Institutes of Health, Bethesda, MD) to detect any relationship between rhizosphere acidification in vitro and seedling size when progeny of the same family were grown in a greenhouse. Ultimately, we sought to identify seedling deciduous azaleas with improved $\mathrm{pH}$ adaptability for use as progenitors of future cultivars.

\section{Materials and Methods}

Germplasm. Four maternal and two paternal parents were used to create seven fullsib families (Table 1). Based on pedigree records, the parents were not related through kinship. All of the parents used to develop the factorial design families were advanced, numbered selections from the University of Minnesota deciduous azalea breeding program, including progenitors and named selections in the Lights series (Hokanson, 2010; Hokanson et al., 2015). We harvested mature seed capsules $\approx 5$ months after crossing and subsequently stored them in a seed cooler at $4{ }^{\circ} \mathrm{C}$ for 6 months.

In vitro culture. Following capsule storage and dehiscence, all seed was surface sterilized for $30 \mathrm{~min}$ in a $2.5 \%$ Plant Preservative Mixture (PPM) (Plant Cell Technology, Inc., Washington, DC) solution using a magnetic stirrer. After rinsing seeds in distilled water, the seeds were plated on petri dishes (35 per plate) containing $20 \mathrm{~mL}$ of Woody Plant Medium with nutrients at half strength (McCown and Lloyd, 1981) (pH 5.7) and $10 \mu \mathrm{L}$ of PPM sterilant. Germination occurred under fluorescent lighting (Sylvania 34W E3e4 bulbs; Osram Sylvania, Inc., Mississauga, CA) with a 24-h photoperiod at a room temperature of $\approx 24{ }^{\circ} \mathrm{C}$. Following germination, the photoperiod duration of the fluorescent lighting was shortened to $16 \mathrm{~h}$ for 2 months as seedlings developed root systems and mature leaves. Screening for rhizosphere acidification began $\approx 8-9$ weeks following

Table 1 . Seven families and the total number of seedlings per family grown in vitro for rhizosphere acidification screening and in the greenhouse for leaf area measurements.

\begin{tabular}{lcc}
\hline \multicolumn{3}{c}{ Number of seedlings used for experiments (in vitro/greenhouse) } \\
\hline & \multicolumn{3}{c}{ Paternal parent } \\
\cline { 2 - 3 } Maternal parent & UMN AZ 223 & UMN AZ 376 \\
\hline UMN AZ 180 & $57 / 171$ & $43 / 99$ \\
UMN AZ 181 & $32 / 90$ & $45 / 171$ \\
UMN AZ 493 & $39 / 45$ & - z \\
UMN AZ 546 & $28 / 81$ & $43 / 99$ \\
\hline
\end{tabular}
$\mathrm{cm}$ in height. The screening medium comprised half-strength Woody Plant Medium adjusted to $\mathrm{pH} 7.8$ with sodium hydroxide and supplemented with 0,310 , or $610 \mathrm{mg} \cdot \mathrm{L}^{-1}$ $\mathrm{CaCO}_{3}$ in the medium to assess the effect of lime concentration on rhizosphere $\mathrm{pH}$ changes.

To visualize $\mathrm{pH}$ change, the $\mathrm{pH}$ indicator phenolsulfonphthalein (phenol red) was incorporated into the tissue culture medium at a final concentration of $90 \mu \mathrm{M}$ before autoclaving. Ten milliliters of the previously described medium, including the phenol red, was poured into $100-\mathrm{mL}$ Pyrex glass tubes. Individual plants from each family were transferred into these tubes that were then sealed with plastic caps and parafilm (Bemis NA, Neenah, WI). The tubes were organized in a completely randomized design under fluorescent lighting with a 16 -h photoperiod throughout the screening process. The temperature of the culture room was maintained at $24{ }^{\circ} \mathrm{C}$ throughout the germination and screening periods.

Greenhouse culture. Seeds from all families were sown in a greenhouse on sphagnum moss in 5 " $\times 5$ " plastic flats under fluorescent lighting with an 18-h photoperiod and daily misting. Greenhouse temperatures were maintained at $27{ }^{\circ} \mathrm{C}$ during the day and $21{ }^{\circ} \mathrm{C}$ at night. Once the seedlings developed mature leaves, they were fertilized once with a $200-p p m$ Peters Excel $21 \mathrm{~N}-2.2 \mathrm{P}-16.4 \mathrm{~K}$ (21-5-20) fertilizer solution (Grace-Sierra CO., Milipitas, CA). The seedlings grew in the plastic flats on sphagnum moss for 10 weeks, after which they were transferred to new plastic flats containing azalea growing medium $(60 \%$ half-inch sieved pine bark and $40 \%$ peat) amended with $0 \%, 10 \%$, or $20 \%$ powdered $\mathrm{CaCO}_{3}$ by dry weight to achieve final medium $\mathrm{pH}$ levels of 5.7, 7.0, and 7.5. The growing medium was saturated with the Peters Excel solution mentioned previously on transplanting. The $\mathrm{pH}$ of the greenhouse medium was measured at the conclusion of the 4-week screening period. Medium $\mathrm{pH}$ for each of the treatments at the end of the screening period $(5.7,7.0$, and 7.5) was determined by creating a $1: 1$ medium to water slurry that was stirred and then allowed to equilibrate for $15 \mathrm{~min}$. The $\mathrm{pH}$ of the slurry was measured using a Mettler-Toledo SevenMulti pH meter (Mettler-Toledo LLC, Columbus, $\mathrm{OH}$ ) at the University of Minnesota Soil Testing Laboratory (St. Paul, MN).

Image analysis protocol (in vitro). Phenol red changes from red to yellow as the initial germination when seedlings were $\approx 1$ 
medium solution becomes more acidic between $\mathrm{pH} 7.5$ and 5.5. To relate the hue measurements of the culture medium to approximate $\mathrm{pH}$, we prepared a series of Pyrex tubes containing tissue culture medium of known $\mathrm{pH}$ containing the phenol red indicator that served as a standard scale. The Pyrex tubes containing progeny from the mating designs were photographed in a windowless room under fluorescent lighting (GE T8 Ultramax Eco XL 28W bulbs; General Electric, Fairfield, CT) using a Canon PowerShot G16 digital camera, with a 6.1-30.5 mm lens (Canon, Inc., Melville, NY), mounted on a tripod. Camera settings included an exposure time of $1 / 8 \mathrm{~s}$, an International Standards Organization (ISO) speed of 80 , a focal length of $6 \mathrm{~mm}$, and a maximum aperture of 1.68 .

Each culture tube in the experiment was photographed for analysis when the seedling was first transplanted and 3 weeks later, with the images saved in JPEG format. To assess changes in medium $\mathrm{pH}$, the images were processed using an ImageJ macro that isolated and cropped the tissue culture medium picture to a $2 \mathrm{~cm} \times 2 \mathrm{~cm}$ square image from the center of the tube (Susko, 2016a). A MATLAB (MathWorks, Inc., Natick, MA) script was used to quantify changes in the color of the phenol red $\mathrm{pH}$ indicator over the 3-week screening period (Susko, 2016b). Our script calculated the average hue, saturation, and value measurements for all pixels in the image. All hue, saturation, and value measurements were reported as decimal values ranging from 0.0 to 1.0 , indicating percentage of degrees around the color wheel for hue, percentage of light for saturation, and percentage of darkness for the value. These values for each image were written to a .csv file by the MATLAB script (Susko, 2016b) for subsequent analyses. We present hue results in the context of the color wheel with a hue value of 0 representing pure red $(\mathrm{pH}$ value $>7.5$ ) and numerical increases toward one indicating a change to orange ( $\mathrm{pH}$ value 7.5 6.0 ) and yellow ( $\mathrm{pH}$ value $<6.0$ ). The change in hue represented change in $\mathrm{pH}$, with an increase in hue indicating acidification of the medium.

Image analysis protocol (greenhouse). Photos were taken of seedlings after transplanting and 4 weeks later to assess the effects of the liming treatments on seedling leaf area. Images of seedling flats were taken using a Nikon D2X digital camera (Nikon Inc., Los Angeles, CA) mounted on a custombuilt light box that maintained consistent lighting for uniform camera exposures. The light box was illuminated with four compact fluorescent bulbs (26 W; General Electric, Fairfield, CT). Manual camera settings (f-stop of $\mathrm{f} / 4$, exposure time of 1/200 s, an ISO speed of 200 , focal length of $35 \mathrm{~mm}$, and a maximum aperture of 1.6) were maintained for all pictures. Images were cropped using ImageJ macros to first reveal the seedling flat and then to divide each flat into 25 separate, uniformly sized sections so that the leaf area change of individual seedlings could be assessed (Fig. 1). Seedlings were distinguished
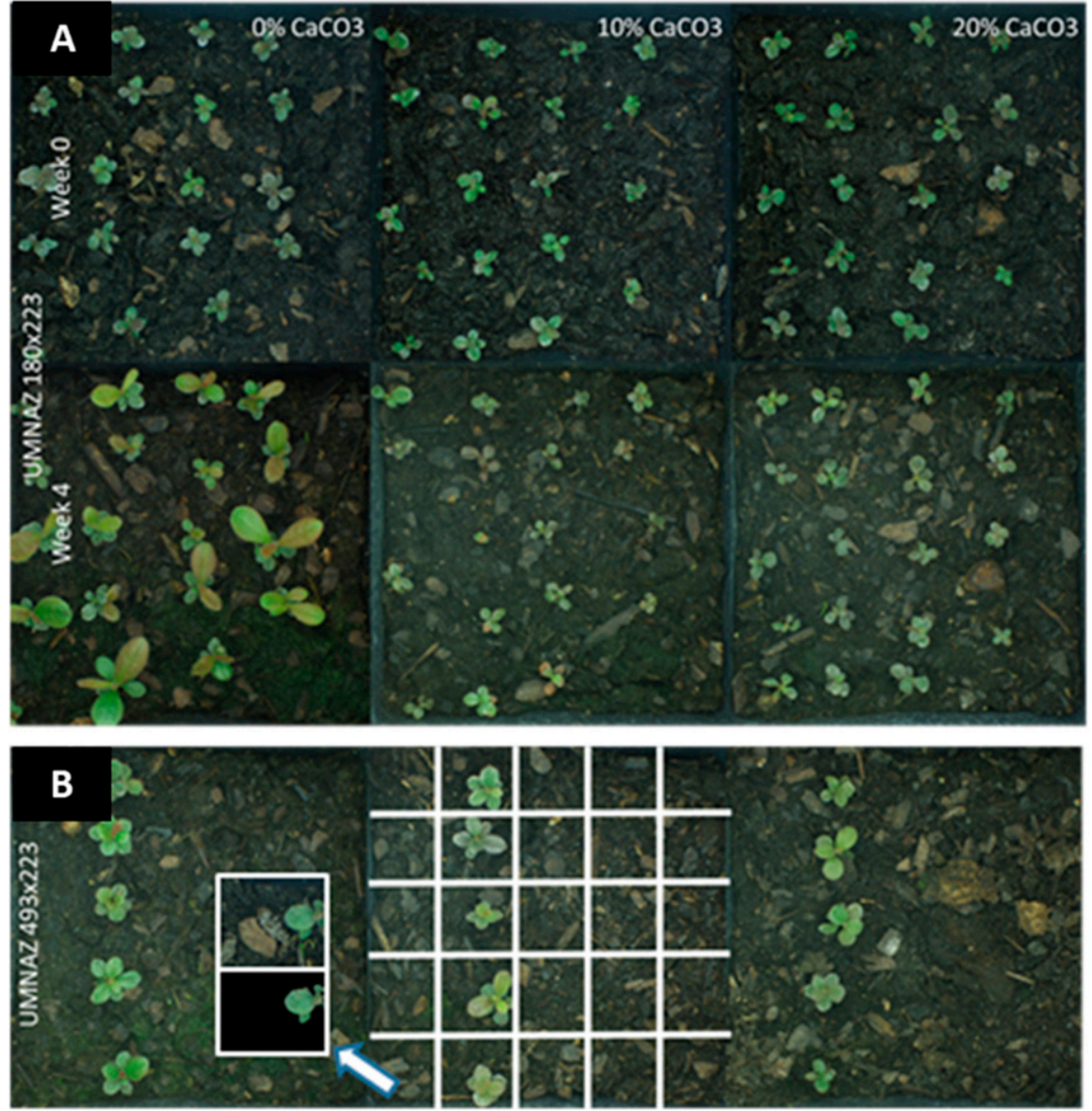

Fig. 1. Images used and processed for phenotyping greenhouse-grown seedlings. (A) Liming treatment designations are displayed across the top, whereas the parental cross and week photos that were taken are displayed along the left side. (B) A grid is superimposed over one seedling flat to illustrate the ImageJ cropping step. An inset image (lower left, noted with arrow) illustrates the MATLAB script used to mask out the greenhouse medium, top square unmasked and bottom masked.

from the medium by contrasting values and hues detected using a MATLAB script, which was initially optimized to detect seedling leaf tissue using the Image Thresholding tool within the MATLAB Image Processing Toolbox (Fig. 1) (Susko, 2016c). Specifically, only pixels with a value measurement between 0.36 and 1.0 and a hue measurement between 0.1 and 0.9 , which represented green leaf surface, were kept for analysis, with all other pixels filtered out of the image because they either constituted greenhouse medium or severely discolored leaf tissue. The cropped images of individual seedlings were then analyzed in MATLAB to compute the leaf area of the seedlings $\left(\mathrm{cm}^{2}\right)$ by scaling the resolution of the image against a known distance. We then computed the change in seedling leaf area by comparing the initial and week 4 images.

Statistics. All variance estimates were calculated using R version 3.3.2 (R Foundation for Statistical Computing, Vienna, Austria), using a linear model and analysis of variance (ANOVA) to compare genotypic and treatment effects among progeny. For the in vitro experiments, phenotypic means for medium hue change were averaged separately by treatment and then averaged by family across the three liming treatments and reported as a percentage of the color wheel $\left(360^{\circ}\right)$, with significance determined using a least significant difference test implemented using the R package Agricolae (De Mendiburu, 2014). We then regressed the change in leaf area for seedlings for each greenhouse liming treatment on seedlings grown in vitro and in the greenhouse by taking the average phenotypic value for each treatment in the greenhouse and in vitro experiments. This was performed to visualize the relationship between in vitro hue change and leaf area change in limed soil-grown plants and to measure the variation in seedling leaf area change accounted for by rhizosphere acidification.

Phenotypic and genetic variances for rhizosphere acidification were estimated using a factorial (partial diallel) crossing scheme. We estimated the narrow-sense heritability for rhizosphere acidification within mating designs as the proportion of progeny variance over the total genetic variance across the three liming treatments. We also estimated the significance of the maternal and paternal effect on the phenotype using a linear model with maternal and paternal genotype factors for hue change (rhizosphere acidification). All genetic variance estimations were made using the mean squares 
generated from the ANOVA on linear models by first estimating the full-sib progeny variance for the factorial design (Eq. 1) to calculate the narrow-sense heritability (Eq. 2) (Bernardo, 2010).

$$
\begin{aligned}
V_{A}= & 2 V_{\text {progeny }} \\
= & 2\left(\frac{\left(\mathrm{MS}_{\text {Females }}-\mathrm{MS}_{\text {Females } \times \text { Treatment }}\right)}{n_{\text {Treatment }}}\right) \\
& h^{2}=\left(\frac{V_{\text {progeny }}}{\left.V_{\text {progeny }}+\frac{\mathrm{MS}_{\text {Family } \times \text { Treatment }}}{n_{\text {Treatment }}}\right)}\right.
\end{aligned}
$$

\section{Results}

In vitro rhizosphere acidification. Depending on the numbers available, between 28 and 57 seedlings per family were screened in vitro and 45-171 seedlings per family were screened in the greenhouse (Table 1). No seed set was obtained from the cross between AZ 493 and AZ 376. We observed rapid germination and initial growth of in vitro seedlings, with most developing at least three mature leaves and branched root systems within 8 weeks after germination. On transferring to high-pH screening medium ( $\mathrm{pH}$ 7.8) amended with $\mathrm{CaCO}_{3}$ treatments, the initial hue values of the medium averaged 0.980 . After 3 weeks, the average hue change was 0.029 across all families and liming treatments tested, indicating a general hue shift from red to orange, and thus a decrease (acidification) in medium $(\mathrm{pH} \approx 6.8-7.0)$. Within each family, average hue change was consistently highest in the $0 \mathrm{mg} \cdot \mathrm{L}^{-1} \mathrm{CaCO}_{3}$ treatment (Table 2). The family UMN AZ $181 \times$ UMN AZ 376 had the lowest observed hue change at 0.024 $(\mathrm{pH} \approx 7.0)$, whereas the family UMN AZ $493 \times$ UMN AZ 223 had the highest observed hue change at $0.050(\mathrm{pH} \approx 6.6)$ (Table 2).

The effect of $\mathrm{CaCO}_{3}$ was significant on observed hue change in vitro, with seedlings placed into the 305 and $610 \mathrm{mg} \cdot \mathrm{L}^{-1} \mathrm{CaCO}_{3}$ medium showing lower measured acidification of the medium (Table 3) (Fig. 2). Comparing family mean phenotypic values averaged across liming treatments revealed significant differences in hue change, with the family $493 \times 223$ showing a significantly higher level of hue change across liming treatments $(P<0.05)$ (Table 2$)$. The maternal genotype effect on $\mathrm{pH}$ change was significant $(P=0.02)$, whereas the paternal genotype effect was not significant $(P=0.94)$ (Table 3 ). Using the progeny variance estimations from phenotypic values in Eqs. [1] and [2], we estimated a narrow-sense heritability $\left(h^{2}\right)$ across families for hue change of 0.38 .

Greenhouse screening. Germination and initial growth of greenhouse-grown seedlings resulted in well-branched root systems and at least three mature leaves on each seedling before screening on liming treatments. Although progeny in each family differed slightly in foliage color before initiating

Table 2. Average tissue culture hue change values for each family and treatment $\left(\mathrm{mg} \cdot \mathrm{L}^{-1} \mathrm{CaCO}_{3}\right)$, with resulting approximate $\mathrm{pH}$ based on colorimetric assay (in parentheses). Initial culture $\mathrm{pH}$ was 7.8.

\begin{tabular}{|c|c|c|c|}
\hline \multicolumn{4}{|c|}{ Avg hue change by family } \\
\hline \multirow[b]{2}{*}{ Maternal parent } & \multicolumn{3}{|c|}{ Paternal parent } \\
\hline & Treatment & UMN AZ 223 & UMN AZ 376 \\
\hline \multirow[t]{4}{*}{ UMN AZ 180} & $0 \mathrm{mg} \cdot \mathrm{L}^{-1} \mathrm{CaCO}_{3}$ & 0.058 & 0.025 \\
\hline & 305 “"z & 0.019 & 0.040 \\
\hline & 610 “" & 0.015 & 0.010 \\
\hline & Mean & $0.031 \mathrm{~b}^{\mathrm{y}}(7.0)$ & $0.025 \mathrm{~b}(7.0)$ \\
\hline \multirow[t]{4}{*}{ UMN AZ 181} & $0 \mathrm{mg} \cdot \mathrm{L}^{-1} \mathrm{CaCO}_{3}$ & 0.052 & 0.035 \\
\hline & 305 “" & 0.009 & 0.014 \\
\hline & 610 “" & 0.015 & 0.021 \\
\hline & Mean & $0.025 \mathrm{~b}(7.0)$ & $0.024 \mathrm{~b}(7.0)$ \\
\hline \multirow[t]{4}{*}{ UMN AZ 493} & $0 \mathrm{mg} \cdot \mathrm{L}^{-1} \mathrm{CaCO}_{3}$ & 0.091 & $-\mathrm{x}$ \\
\hline & 305 “" & 0.033 & \\
\hline & 610 “" & 0.025 & \\
\hline & Mean & 0.050 a $(6.6)$ & \\
\hline \multirow[t]{4}{*}{ UMN AZ 546} & $0 \mathrm{mg} \cdot \mathrm{L}^{-1} \mathrm{CaCO}_{3}$ & 0.043 & 0.040 \\
\hline & 305 “" & 0.038 & 0.027 \\
\hline & $610 “ "$ & 0.012 & 0.035 \\
\hline & Mean & $0.030 \mathrm{~b}(7.0)$ & $0.034 \mathrm{ab}(7.0)$ \\
\hline
\end{tabular}
Values were then averaged across the three liming treatments to obtain an average hue change per family.

${ }^{\mathrm{z}} \mathrm{mg} \cdot \mathrm{L}^{-1}$ of $\mathrm{CaCO}_{3}$ liquid tissue culture medium.

${ }^{y}$ Any two hue change family means not followed by the same letter are significantly different at $P<0.05$. ${ }^{\mathrm{x}}$ Capsules lost from cross.

Table 3. Analysis of variance results showing the paternal, maternal, and treatment effects on hue detected in vitro.

\begin{tabular}{lrcrr}
\hline Source of variation & df & Mean squares & $F$ value & $P$ value \\
\hline Maternal $(M)$ & 3 & 0.00444 & 3.340 & 0.021 \\
Paternal $(P)$ & 1 & 0.00001 & 0.006 & 0.938 \\
$\mathrm{CaCO}_{3}$ treatment $(T)$ & 2 & 0.01741 & 13.087 & $<0.001$ \\
$M \times P$ & 2 & 0.00004 & 0.031 & 0.969 \\
$M \times T$ & 6 & 0.00275 & 2.070 & 0.059 \\
$P \times T$ & 2 & 0.00123 & 0.921 & 0.400 \\
$M \times P \times T$ & 4 & 0.00197 & 1.477 & 0.211 \\
Residuals & 178 & 0.00133 & & \\
\hline
\end{tabular}

high-pH/CaCO${ }_{3}$ treatments, the MATLAB image analysis scripts were successful in differentiating healthy, primarily green leaf tissue from the greenhouse medium background. This enabled us to quantify the area of green leaf tissue for each family at two time points. Mean initial green leaf areas were uniform for most families in the experiment, with only UMN AZ $180 \times 223$ and $181 \times 376$ having a significantly lower mean initial green leaf area (Fig. 3). Following the 4-week screening period, the effect of family on green leaf area change was highly significant across liming treatments $(P<0.001)$, with visible trends in seedling green leaf area apparent across families and liming treatments (Fig. 4). After 4 weeks, seedlings in the $10 \%$ and $20 \% \mathrm{CaCO}_{3}$ by weight treatments were observed to have reduced leaf area and reddened or necrotic leaf tissue $(P<0.001)$ (Table 4; Fig. 1).

The $10 \%$ and $20 \%$ lime by weight treatments resulted in seedlings with lower and sometimes negative leaf area change (Fig. 4). This negative leaf area change indicates a reduction in the amount of green tissue over the screening period due to leaf death and/or severe discoloration which caused leaf hue to fall into the threshold of the image that was masked by the MATLAB analysis.
In comparing the average green leaf area change per treatment in the greenhouse with in vitro hue change in a given liming treatment for each family, in vitro hue change was positively correlated with green leaf area measured in the greenhouse (Fig. 5). In a linear model with in vitro hue change as the independent and greenhouse green leaf area as the dependent variable, the calculated $R^{2}$ value was 0.45 .

\section{Discussion}

This method of quantifying relative changes in rhizosphere $\mathrm{pH}$ may allow breeders of woody plants to more easily identify genotypes with superior capacity for iron uptake on high-pH or calcareous media. Although colorimetric visualization of changes in medium $\mathrm{pH}$ has been used in vitro for some time (Marschner et al., 1982; $\mathrm{Yi}$ and Guerinot, 1996), this experiment represents the phenotyping potential of such an approach in a high-throughput, quantitative breeding context. Although variation among families for rhizosphere acidification was significant, it is important to note the broad reduction in rhizosphere acidification across families growing in medium containing $\mathrm{CaCO}_{3}$. This reduction in acidification in the presence of $\mathrm{CaCO}_{3}$ is due to the buffering 


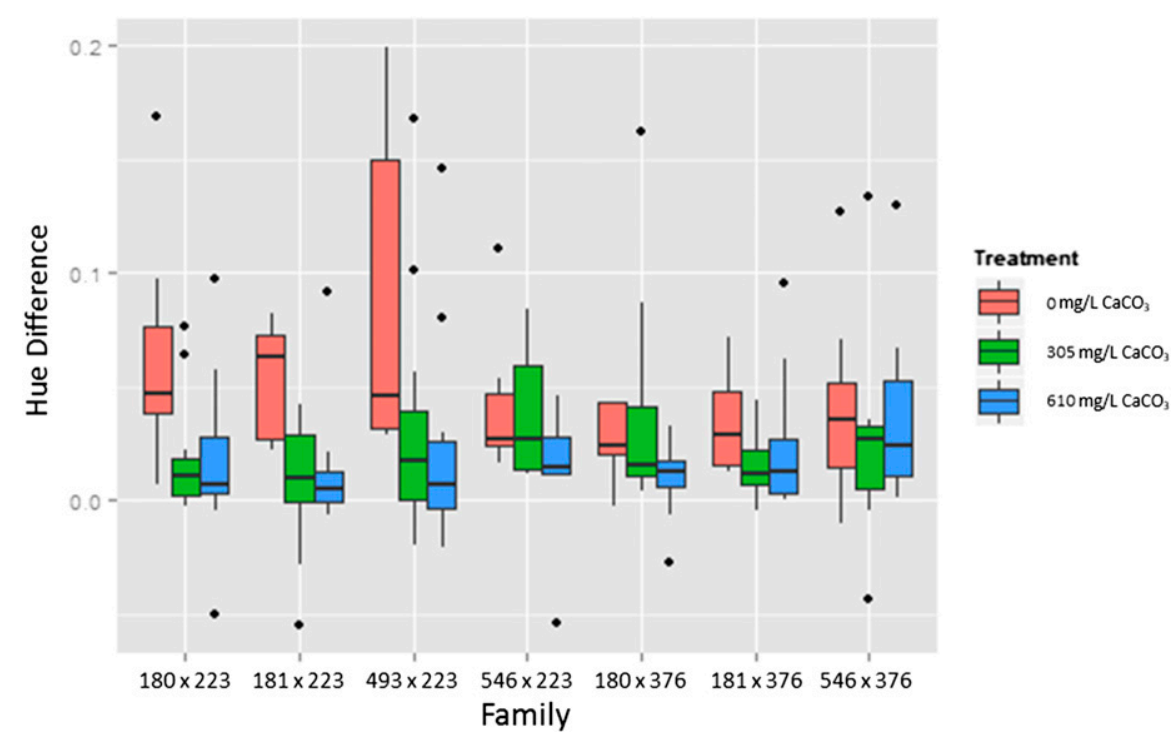

Fig. 2. Boxplot depicting differences in hue resulting from $\mathrm{CaCO}_{3}$ concentration in in vitro-grown seedlings. Whereas there were significant differences between the control $\left(0 \mathrm{mg} \cdot \mathrm{L}^{-1} \mathrm{CaCO}_{3}\right)$ and the $\mathrm{CaCO}_{3}$ treatments $(P<0.001)$, the difference in mean hue between the two $\mathrm{CaCO}_{3}$ treatments was not significant.

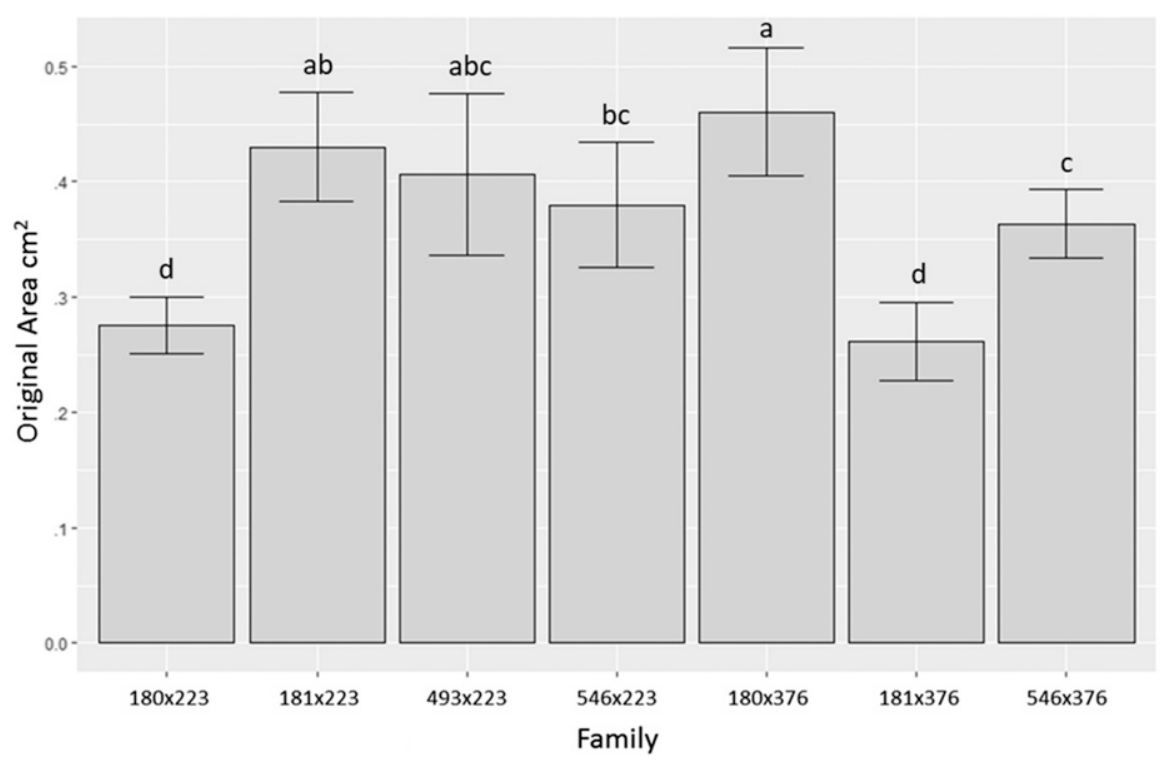

Fig. 3. Bar graph showing the original green leaf area mean and $95 \%$ confidence intervals for seedlings from each family used in the greenhouse phenotyping experiment. Unique letter combinations indicate a significant difference $(P<0.05)$ between family mean original seedling leaf areas.

effect of bicarbonate ions on $\mathrm{pH}$ (Brady and Weil, 2004; Briat and Lobréaux, 1997). Thus, $\mathrm{CaCO}_{3}$ limits total acidification of soil, and thus the ability of rhizosphere acidification to increase the solubility of micronutrients such as iron. Deciduous azalea intolerance to $\mathrm{CaCO}_{3}$ has long been documented (Hume, 1948), and it is likely that the buffering of rhizosphere acidification contributes in part to the poor performance of deciduous azaleas on these soils. Nonetheless, quantifiable genetic variation for rhizosphere acidification and its positive relationship with seedling leaf area change suggest that genetic variation for alkalinity tolerance exists among deciduous azalea genotypes.

The narrow-sense heritability value of 0.38 for rhizosphere acidification we observed unlikely scenario for a trait with such continuous variation (Bernardo, 2010), the inclusion of more paternal parents in a mating design will likely show a significant paternal effect on rhizosphere acidification variation.

Although increase in hue measured in vitro over time suggests that rhizosphere acidification occurs in deciduous azaleas, it does not confirm that it is a direct response to iron-limited conditions present in high-pH or calcareous soils. The rhizosphere acidification response in dicot plants (Strategy 1) is typically initiated in response to low iron in tissues and involves proton extrusion through reverse ATPase (Guerinot and Yi, 1994). Among woody genera, this direct rhizosphere acidification response has been confirmed in Quercus L., Vitis L., and Prunus L. (Gogorcena et al., 2001; Gonzalo et al., 2011; Ksouri et al., 2006), whereas it is absent in Vaccinium L. under iron-deficient culture medium in the absence of $\mathrm{CaCO}_{3}$ (Nunez et al., 2015). The significant reduction in rhizosphere acidification in the presence of increased levels of $\mathrm{CaCO}_{3}$ we observed across all deciduous azalea families was similar to that shown previously among Vitis L. cultivars (Ksouri et al., 2006). The observed acidification in our experiment could also have been indirectly caused by the uptake of ammonium cations, which were present in the Woody Plant Medium used. Active extrusion of protons is also a mechanism used by root cells to stabilize intracellular charge balances that facilitate cation uptake such as ammonium (Haynes, 1990). This subsequently acidifies the rhizosphere and occurs independently of proton extrusion due to iron deficiency (Escobar et al., 2006). An additional experiment looking at acidification in the absence of ammonium at high $\mathrm{pH}$ would be necessary to confirm a physiological basis for direct acidification of the rhizosphere under iron-limited conditions present in calcareous or high-pH media.

The reduced change in leaf area among greenhouse-grown seedlings in treatments with $\mathrm{CaCO}_{3}$ relative to the treatment lacking $\mathrm{CaCO}_{3}$ further demonstrates the effect of high $\mathrm{pH}$ stress induced by bicarbonate ions on deciduous azalea seedlings. In addition, the effect of family on greenhouse seedling leaf area change was also significant. This pattern of treatment and family differences in response to increased $\mathrm{CaCO}_{3}$ mirrored that of the in vitro rhizosphere acidification experiment. Full-sib seedlings with high rhizosphere acidification on average had greater leaf areas when grown in greenhouse liming treatments. Ideally, the $\mathrm{pH}$ of the greenhouse control treatment would have been high, but this was not feasible to maintain throughout the 4-week experiment. Nonetheless, this greenhouse medium treatment lacking $\mathrm{CaCO}_{3}$ serves as a useful point to correlate leaf area change with rhizosphere acidification in the absence of bicarbonate buffering ions. Suppression of rhizosphere acidification within this deciduous azalea breeding population in the presence 


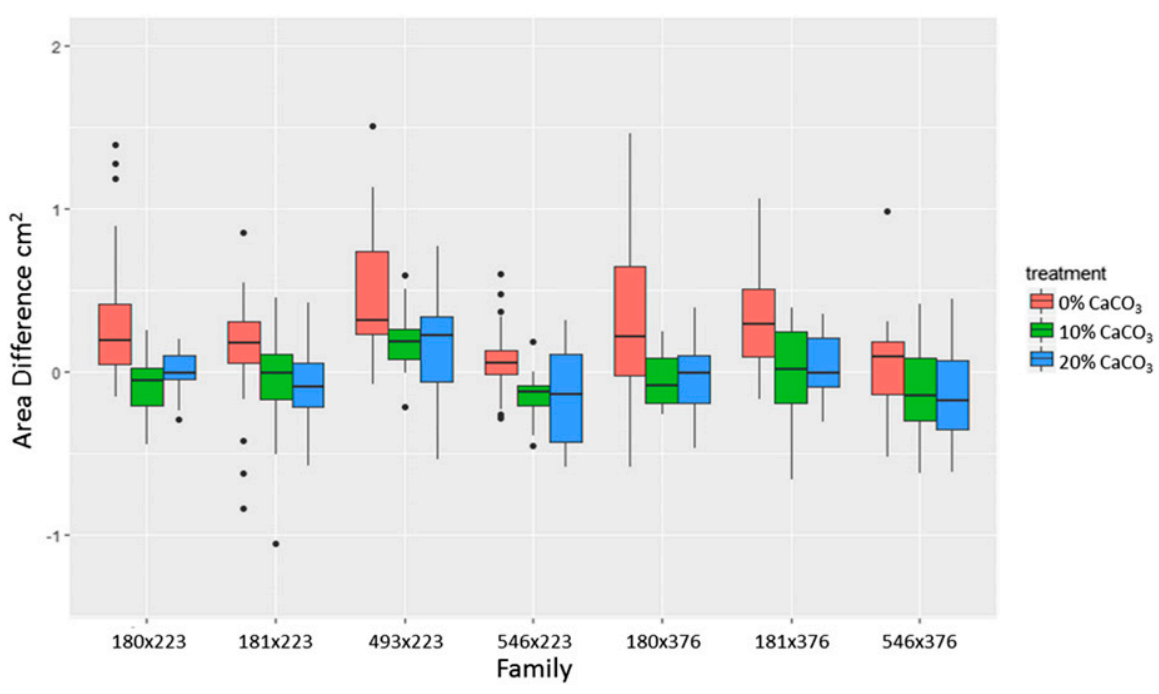

Fig. 4. Change in leaf area $\left(\mathrm{cm}^{2}\right)$ of seedlings in seven families growing in three $\mathrm{CaCO}_{3}$ treatments in the greenhouse over 4 weeks, measured through MATLAB image analysis. The effect of the liming treatments significantly reduced seedling leaf areas $(P<0.001)$.

Table 4. Analysis of variance (ANOVA) results for seedling leaf area change in the greenhouse liming experiment. Maternal and paternal effects are not separated in this ANOVA and are regarded together as the factor "Family."

\begin{tabular}{lrccr}
\hline Source of variation & df & Mean squares & $F$ value & $P$ value \\
\hline Family & 6 & 1.004 & 9.031 & $<0.001$ \\
Treatment & 2 & 0.883 & 7.948 & $<0.001$ \\
Family $\times$ treatment & 12 & 0.903 & 8.127 & $<0.001$ \\
Residuals & 949 & 0.111 & & \\
\hline
\end{tabular}

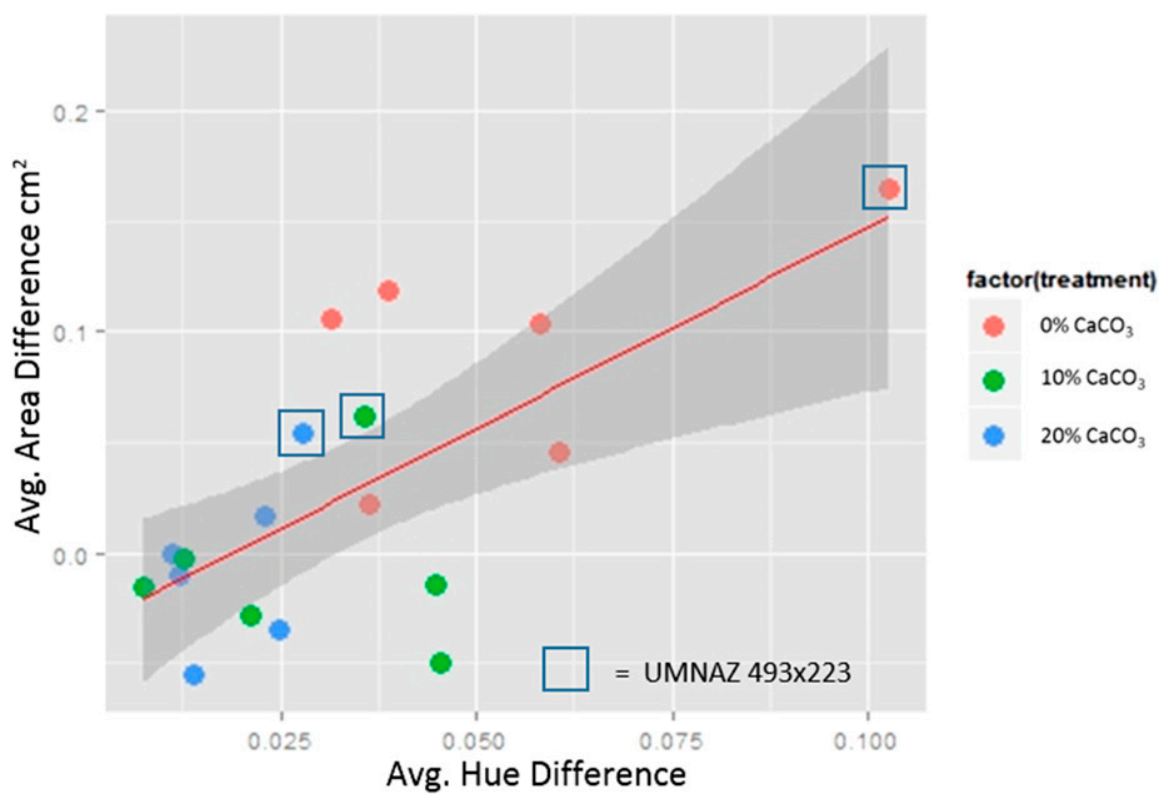

Fig. 5. Relationship between in vitro average final hue for each treatment and family and greenhouse seedling green leaf area for each treatment and family. The dark shading around the regression line $\left(R^{2}=0.45\right)$ denotes a $95 \%$ confidence interval. Individual dots represent a treatment average for each family, with treatments coded by color. The family UMNAZ $493 \times 223$ seedlings in the $10 \%$ and $20 \%$ $\mathrm{CaCO}_{3}$ treatments, where average final hue explained significantly more than $45 \%$ of the variation in leaf area change on calcareous greenhouse media, are denoted by blue boxes.

of bicarbonate ions reduces seedling growth, and the variability in response among full-sib families suggests a genetic component to tolerance of high-pH, calcareous media.

Regardless of the exact mechanism by which we observed a $\mathrm{pH}$ change in the medium, the decrease in $\mathrm{pH}$ would nonetheless have consequences for iron solubility at the root-soil interface. The genotype specific variation, measurable narrow-sense heritability, and positive relationship between rhizosphere acidification and leaf area increase suggest that selection for in vitro hue change could serve as a means to improve $\mathrm{pH}$ adaptability in deciduous azalea. It is unknown at this point the degree of rhizosphere acidification that will occur in mature plants derived from this breeding population as mature plants will likely be subject to stronger genotype by environment interactions and genotype by year interactions that affect relevant phenotypes for $\mathrm{pH}$ adaptability. Determining mature plant response to elevated $\mathrm{pH}$ and high calcareous soils will require longer term observation of mature plants in field experiments. This could be accomplished via a multiyear observation of deciduous azalea growth rates under high $\mathrm{pH}$ stress and might shed light on the relationship between rhizosphere acidification in seedlings and the performance of genotypes in stressful landscape situations over time.

The high-throughput phenotyping of seedling leaf area change and rhizosphere acidification enabled data collection with relative speed in a nondestructive way, with methods readily transferable to future studies using the scripts presented here. Given market demand for a steady flow of new ornamental landscape cultivars, there is an incentive to accelerate the timeframes for evaluating plant germplasm. Speeding up the breeding process will require more efficient high-throughput phenotyping protocols (Van Nocker and Gardiner, 2014). Speed and accuracy, both of which will enable timely selection of azalea cultivars with improved $\mathrm{pH}$ adaptability, could be greatly improved through the use of image-based phenotyping (Furbank and Tester, 2011). The automated nature of image analysis through scripts removes potential ratings bias and saves substantial time. The scalability of image-based phenotyping to higher numbers of progeny also allows more data to be collected for less effort, which could lead to improved estimates of quantitative trait parameters and response to selection in breeding populations (Bernardo, 2010).

Broadly speaking, these methods should prove useful for quickly screening new germplasm, and when coupled with the use of a mating design, can estimate progeny variation for both rhizosphere acidification and seedling leaf area change. This will allow for estimations of quantitative trait parameters important for $\mathrm{pH}$ adaptability that were previously difficult to obtain for a woody plant. Such systematic application could be applied in Rhododendron to identify germplasm sources to develop durable cultivars for broader landscape use.

\section{Literature Cited}

Berger, B., B. Parent, and M. Tester. 2010. Highthroughput shoot imaging to study drought responses. J. Expt. Bot. 61:3519-3528.

Bernardo, R. 2010. Estimating genetic variances, $p$. 147-176. In: Breeding for quantitative traits in plants. Stemma Press, Woodbury, MN

Brady, N.C. and R. Weil. 2004. Elements of the nature and properties of soils. 2nd ed. Pearson Prentice Hall, Upper Saddle River, NJ.

Briat, J-F. and S. Lobréaux. 1997. Iron transport and storage in plants. Trends Plant Sci. 2:187-193. 
Clark, R.T., A.N. Famoso, K. Zhao, J.E. Shaff, E.J. Craft, C.D. Bustamante, S.R. McCouch, D.J. Aneshansley, and L.V. Kochian. 2013. Highthroughput two-dimensional root system phenotyping platform facilitates genetic analysis of root growth and development. Plant Cell Environ. 36:454-466.

De Mendiburu, F. 2014. Agricolae: Statistical procedures for agricultural research. R. package, version 1, p. 1-6.

Dunemann, F., R. Kahnau, and I. Stange. 1999. Analysis of complex leaf and flower characters in Rhododendron using a molecular linkage map. Theor. Appl. Genet. 98:1146-1155.

Escobar, M.A., D.A. Geisler, and A.G. Rasmusson. 2006. Reorganization of the alternative pathways of the Arabidopsis respiratory chain by nitrogen supply: Opposing effects of ammonium and nitrate. Plant J. 45:775-788.

Furbank, R.T. and M. Tester. 2011. Phenomicstechnologies to relieve the phenotyping bottleneck. Trends Plant Sci. 16:635-644.

Froechlich, D.M. and W.R. Fehr. 1981. Agronomic performance of soybeans with differing levels of iron deficiency chlorosis on calcareous soil. Crop Sci. 21:438-441.

Galle, F.C. 1974. Azaleas. Oxmoor House, Inc., Birmingham, AL.

Gonzalo, M.J., M.A. Moreno, and Y. Gogorcena. 2011. Physiological responses and differential gene expression in Prunus rootstocks under iron deficiency conditions. J. Plant Physiol. 168:887-893.

Gogorcena, Y., N. Molias, A. Larbi, J. Abadia, and A. Abadia. 2001. Characterization of the responses of cork oak (Quercus suber) to iron deficiency. Tree Physiol. 21:1335-1340.

Guerinot, M.L. and Y. Yi. 1994. Iron: Nutritious, noxious, and not readily available. Plant Physiol. 104:815-820.
Haynes, R.J. 1990. Active ion uptake and maintenance of cation-anion balance: A critical examination of their role in regulating rhizosphere pH. Plant Soil 126:247-264.

Hokanson, S.C. 2010. "Lights" in the land of 10,000 lakes. In: Rhododendrons, camellias and magnolias. Royal Horticultural Society, London, UK.

Hokanson, S. C., S. T. McNamara, N. Rose, K. Zuzek, and H. Pellett. 2015. 'UMNAZ 493' and 'UMNAZ 502'; two new cold hardy deciduous azalea selections from the University of Minnesota. HortScience 50S:053 (abstr.).

Hume, H.H. 1948. Azaleas: Kinds and culture. The Macmillan Company. New York, NY.

Ksouri, R., S. M'rah, M. Gharsalli, and M. Lachaal. 2006. Biochemical responses to true and bicarbonate-induced iron deficiency in grapevine genotypes. J. Plant Nutr. 29:305-315.

Marschner, H., V. Römheld, and H. OssenbergNeuhaus. 1982. Rapid method for measuring changes in $\mathrm{pH}$ and reducing processes along roots of intact plants. Z. Pflanzenphysiol. 105:407-416.

McCown, B.H. and G. Lloyd. 1981. Woody plant medium (WPM)-a mineral nutrient formulation for microculture of woody plant-species. HortScience 16:453.

Moe, S. and H. Pellett. 1986. Breeding for cold hardy azaleas in the land of the northern lights. J. Amer. Rhododendr. Soc. 40(3).

Nunez, G.H., J.W. Olmstead, and R.L. Darnell. 2015. Rhizosphere acidification is not part of the strategy I iron deficiency response of Vaccinium arboreum and the southern highbush Blueberry. HortScience 50:10641069.

Preil, W. and R. Ebbinghaus. 1994. Breeding of lime tolerant Rhododendron rootstocks. Acta Hort. 364:61-70.
Shi, L., T. Shi, M.R. Broadley, P.J. White, Y. Long, J. Meng, F. Xu, and J.P. Hammond. 2013. High-throughput root phenotyping screens identify genetic loci associated with root architectural traits in Brassica napus under contrasting phosphate availabilities. Ann. Bot. (Lond.) 112:381-389.

Susko, A.Q. 2016. Phenotypic and genetic variation for rhizosphere acidification, a candidate trait for $\mathrm{pH}$ adaptability, in deciduous azalea (Rhododendron sect. Pentanthera). Univ. Minnesota, Twin-Cities, MS Diss.

Susko, A.Q. 2016a. Rhisozphere_crop.txt: An ImageJ script for cropping seedling flats. 4 Mar. 2016 $<$ https://github.com/Hortus/ImageJ_scripts/ $\mathrm{blob} / \mathrm{master} /$ Rhizosphere_Crop.txt $>$.

Susko, A.Q. 2016b. RhisozphereAcidification.m: A MATLAB script for analyzing hue change of in-vitro rhizospheres. 4 Mar. 2016. <https:// github.com/Hortus/MATLAB_Scripts/blob/ master/RhizosphereAcidification.m $>$.

Susko, A.Q. 2016c. SeedlingDetection.m: A MATLAB script for distinguishing seedling leaf tissue from greenhouse media. 4 Mar. 2016. <https://github.com/Hortus/MATLAB_ Scripts/blob/master/SeedlingDetection.m $>$.

Van Nocker, S. and S.E. Gardiner. 2014. Breeding better cultivars, faster: Applications of new technologies for the rapid deployment of superior horticultural tree crops. Hort. Res. 1:14022-14032.

Widrlechner, M. 1982. Studies on the breeding potential and genetics of Hybrid Azalea, Rhododendron $\times$ kosterianum Schneider $\times$ Rhododendron prinophyllum (Small). Univ. Minnesota, Millias., St. Paul, MN, Doctoral Diss.

Yi, Y. and M.L. Guerinot. 1996. Genetic evidence that induction of root Fe (III) chelate reductase activity is necessary for iron uptake under iron deficiency. Plant J. 10:835-844. 